\title{
Adsorption of the Three-phase Emulsion on Various Solid Surfaces
}

\author{
Yasutaka Enomoto ${ }^{*}$, Yoko Imai ${ }^{2}$ and Kazuo Tajima ${ }^{2}$ \\ ${ }^{1}$ Graduate School of Applied Chemistry, Kanagawa University, 3-27-1, Rokkakubashi, Kanagawa-ku, Yokohama-shi, Kanagawa, 221-8686, \\ JAPAN \\ ${ }^{2}$ Project of Three-Phase Emulsification Technology, Kanagawa University, 3-27-1, Rokkakubashi, Kanagawa-ku, Yokohama-shi, Kanagawa, \\ 221-8686, JAPAN
}

\begin{abstract}
The present study investigates the adsorption of the three-phase emulsion on various solid/water interfaces. Vesicles can be used as emulsifiers in the three-phase emulsions and act as an independent phase unlike the surfactant used in conventional emulsions; therefore, it is expected that the three-phase emulsion formed by the adhesion of vesicles to the oil/water interface will adsorb on various solid/water interfaces. The cationic three-phase emulsion was prepared to encourage emulsion adsorption on negatively charged solid substrates in water. The emulsifier polyoxyethylene-(10) hydrogenated castor oil was rendered cationic by mixing with the surfactant cetyltrimethylammonium bromide and then used to prepare the cationic three-phase emulsion of hexadecane-in-water. Three solid substrates (silicon, glass, and copper) were dipped in the cationic emulsion and the emulsion was found to adsorb on the solid substrates while maintaining its structure. The amount of hexadecane adsorbed on the various surfaces was investigated by gas chromatography and found to increase with increasing hexadecane concentration in the emulsion and eventually plateaued just like molecular adsorption. The maximum surface coverage of the emulsion on the substrates was approximately $80 \%$. However, even the equivalent nonionic three-phase emulsion was found to adsorb on the three solid surfaces. This was attributed to a novel mechanism of irreversible adhesion via the van der Waals attractive force.
\end{abstract}

Key words: three-phase emulsion, emulsion adsorption, solid surface, cationic vesicle, hydrogenated castor oil

\section{INTRODUCTION}

Sparingly water-soluble amphipathic substances are known to form various lamellar structures at a suitable temperature. External energy (mechanical power) has been used to form vesicles in aqueous systems ${ }^{1-11)}$. However, we have previously demonstrated the spontaneous transition from lamellar liquid crystals to vesicles without using external energy ${ }^{12)}$.

Much research has been conducted on the properties of vesicles. In particular, the application of vesicles to drug delivery systems has been attempted using a variety of techniques such as the solubilization of oil-soluble materials in the bilayer and the encapsulation of water-soluble materials in the inner water-phase ${ }^{13-18)}$

We found that a stable emulsion was formed by mixing $1-2$ wt $\%$ of vesicles or a lamellar dispersion and 20-50 wt $\%$ oil ${ }^{19)}$. This emulsification uses the irreversible adhesion of nanoparticles and differs completely from conventional emulsification, which uses a decrease in the interfa- cial tension upon molecule adsorption. If hydrophilic nanoparticles can coexist with oil droplets by adhesion to the oil/water interface and form a coating, the oil droplet surface can be changed from hydrophobic to hydrophilic. This phenomenon is called three-phase emulsification ${ }^{20-25)}$. It is possible to observe the shape of the three-phase emulsion directly by TEM, SEM, and AFM. Such analyses have shown that the three-phase emulsions fix on solid surfaces while maintaining their structure ${ }^{26)}$.

If the three-phase emulsions containing functional oils display high adsorbability on solid substrates, this feature could be utilized to improve the medicinal effects and sustain the releasing effects of emulsified oil-soluble agents. Functional oils such as agricultural chemicals and surface treating agents are applied by spraying on an objective interface; increasing the adsorbability of these oils is particularly important for reducing the amounts required and, thus, protecting the environment and reducing costs ${ }^{27-31}$. These problems need to be resolved in various industries.

\footnotetext{
*Correspondence to: Yasutaka Enomoto, Graduate School of Applied Chemistry, Kanagawa University, 3-27-1, Rokkakubashi, Kanagawa-ku, Yokohama-shi, Kanagawa, 221-8686, JAPAN

E-mail: r201470174yp@jindai.jp

Accepted March 10, 2017 (received for review December 22, 2016)

Journal of Oleo Science ISSN 1345-8957 print / ISSN 1347-3352 online

http://www.jstage.jst.go.jp/browse/jos/ http://mc.manusriptcentral.com/jjocs
} 
The present study attempts to verify experimentally whether the three-phase emulsions can adsorb on various solid surfaces. Moreover, we aim to clarify adsorbability differences between the three-phase emulsions and surfactant emulsions.

\section{THEORY OF EMULSION ADSORPTION}

The phenomenon of emulsion adsorption at interfaces has never been reported. This is due to thermodynamic considerations that render surfactant-emulsion adsorption on interfaces unlikely. However, differences in the structure of the three-phase emulsions, compared to traditional surfactant-based emulsions, appear to allow them to adsorb directly at interfaces while maintaining their structure. In this section, we discuss the theoretical possibility of oil/water-type emulsion adsorption at interfaces.

First, we consider the existence of surfactant emulsions and solid substrates in water. The water-soluble nature of surfactants means that every interfacial phase is singular in surfactant-emulsion systems and they can be discussed in terms of the Gibbs' phase rule. By contrast, the nanoparticles in the three-phase emulsion system are an independent phase because of the requirement for them to be insoluble in both water and oil. Therefore, the three-phase emulsion system in which no soluble components exist cannot be discussed in terms of the Gibbs' phase rule. However, the number of phases and contact interfaces in emulsion systems can be counted easily.

There are three phases to consider in the adsorption of surfactant-emulsions on solid surfaces: (1) water, (2) oil, and (3) solid substrate. Therefore, three possible contact interfaces can form: (1) oil/water, (2) water/solid substrate, and (3) oil/solid substrate. In this case, the surfactant molecules adsorbing at the oil/water interface must be in adsorption-desorption equilibrium with a monomer and, moreover, the surfactant component should always coexist in the bulk water phase (even if as a species of self-assemblies such as micelles) and is not an independent component. Therefore, when surfactant emulsions adsorb to solid substrates, the surfactant on the adsorbed face loses the adsorption-desorption equilibrium. In other words, it is thermodynamically impossible that one surfactant molecule simultaneously adsorbs and indicates the same surface activity to both oil/water and water/solid substrate interfaces at the same adsorbed site. As a result, the emulsion/solid substrate interface cannot be formed by adsorption of the emulsion on the solid substrate.

By contrast, there are four phases to consider in the adsorption of the three-phase emulsion systems on solid surfaces: (1) water, (2) oil, (3) nanoparticle, and (4) solid substrate. Therefore, the formation of six different contact interfaces is possible: (1) oil/nanoparticle, (2) water/solid

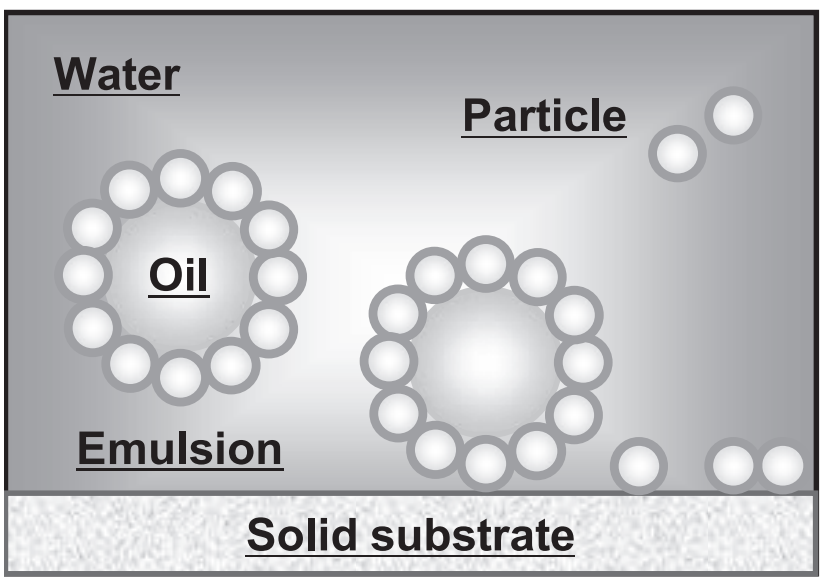

Scheme 1 Illustration of three-phase-emulsion adsorption on a solid substrate.

substrate, (3) water/nanoparticle, (4) oil/solid substrate, (5) oil/water, and (6) nanoparticle/solid substrate. There is no subordinate relationship between each phase in the three-phase emulsion system described above as the nanoparticle is treated as an independent phase. Therefore, a nanoparticle can simultaneously adsorb on both oil/ water and water/solid substrate interfaces at the same site as illustrated in Scheme 1. Thus, the three-phase emulsion can theoretically adsorb directly on a solid substrate while maintaining its structure. The purpose of the present study is to experimentally examine the adsorption of the threephase emulsions with regards to the aforementioned theoretical concepts.

Although the adsorption of nanoparticles on solid substrates in the three-phase emulsion system should be expressed as adhesion, it is hereafter described as adsorption from a phenomenological point of view.

\section{EXPERIMENTAL PROCEDURES}

\subsection{Materials}

Polyoxyethylene-(10) hydrogenated castor oil (HCO-10, MW: 1380) was used as the emulsifier for the three-phase emulsification. Scheme 2 shows the molecular formula of HCO-10. HCO-10 was purchased from Nikko Chemicals Co., Ltd. and was used without further purification. HCO-10 is known to spontaneously form vesicles in water ${ }^{32,33)}$.

Cationizing an emulsion generally increases its adsorbability on a solid substrate. Therefore, a cationic threephase hexadecane-in-water emulsion was prepared using HCO-10 vesicles that were rendered cationic by mixing with the cationic surfactant cetyltrimethylammonium bromide (CTAB, MW: 364.45). A hexadecane-in-water emulsion was also prepared with CTAB alone in order to investigate the absorption of a typical surfactant emulsion. Hexadecane and CTAB were purchased from Wako Pure 


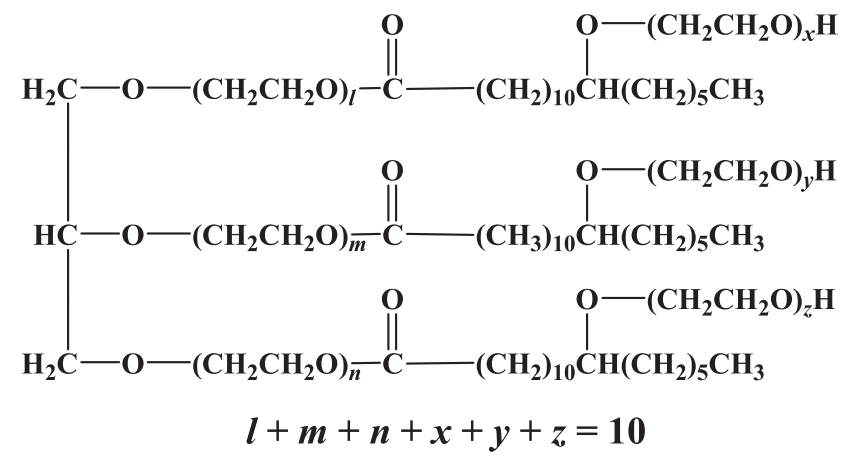

Scheme 2 Structure of polyoxyethylene-(10) hydrogenated castor oil (HCO-10).

Chemical Industries and were used without further purification.

Emulsion adsorption was tested on three different solid substrates: a silicon wafer, glass, and a copper plate. The silicon wafer was purchased from Semitec, glass was purchased from Matsunami Glass, and copper was purchased from Sumitomo Metal Mining. Each surface was cleaned in a chromic acid mixture for 1 day before the adsorption tests. After washing off the chromic acid mixture using water, the solid substrates were used in wet state as soon as possible. The zeta potentials of the solid substrates in water were negative and are presented in Table 1 together with the contact angle of water on each solid surface. Vesicle adsorption was tested on a cellulose fiber surface provided by Hakuto.

\subsection{Apparatus}

The zeta potential of the vesicles, emulsions, and solid substrates were measured using an electrophoretic light scattering photometer(Otsuka Electronics, ELS-8000) at $20^{\circ} \mathrm{C}$. The vesicles and emulsions were diluted with deionized water by 1:10000 for the zeta potential measurements.

The vesicle adsorption was observed at each stage by scanning electron microscopy (SEM, Hitachi, S-4000). Prior to observation, the sample was pretreated according to the following procedure: a sample was mounted on a silicon wafer, spread widely and thinly, dried in a desiccator overnight, and then coated with carbon vapor to diminish electrostatic perturbations. The emulsion shapes were observed using negative-staining transmission electron microscopy (JEOL, JEM-2000EX / FXII) ${ }^{34)}$. Prior to obser-

Table 1 Zeta potentials of the three solid substrates studied here and their contact angles with water.

\begin{tabular}{ccc}
\hline Solid substrates & Zeta potential $/ \mathrm{mV}$ & Contact angle ${ }^{\circ}$ \\
\hline Silicon wafer & -32 & 88.0 \\
Glass & -70 & 6.6 \\
Copper & -100 & 83.0 \\
\hline
\end{tabular}

vation, the emulsions were pretreated using the following procedure: the sample was mounted on a copper mesh and dried in a desiccator overnight at ambient temperature. It is generally difficult to observe organic assemblies by transmission electron microscopy because of their low charge density. Negative staining surrounds the organic assembly with ammonium molybdate, which is non-structural and has a high charge density, thus increasing the contrast between the organic assembly and the background and rendering the assembly shape observable. The entire distribution of the three-phase emulsion adsorbed on siliconwafer and glass surfaces was observed by optical microscopy (KEYENCE, VHX-100F / VH-Z-100).

Gas chromatography (Yokogawa Electric Corporation, HP5890 SERIES II) was used to measure the amount of hexadecane adsorbed on the various surfaces by computing the peak-area ratio between the solubilizing substance and a decane internal standard (a special grade reagent purchased from Wako Pure Chemical Industries).

\subsection{Emulsion preparation}

HCO-10 was first completely dissolved in an appropriate amount of ethanol and CTAB was then added to the HCO-10 ethanol solution. The ethanol was then removed by passing nitrogen gas through the solution to produce a molecularly mixed substance.

The molar fraction of CTAB in the molecularly mixed substance is calculated by Equation (1).

$$
\boldsymbol{X}_{S}=\frac{\boldsymbol{n}_{C T A B}}{\boldsymbol{n}_{H C O-10}+\boldsymbol{n}_{C T A B}},
$$

where $X_{S}$ is the mole fraction of CTAB, and $n_{\mathrm{HCO}-10}$ and $n_{\mathrm{CTAB}}$ are the moles of HCO-10 and CTAB, respectively.

The vesicles were prepared by dispersing $10 \mathrm{wt} \%$ of the resulting $\mathrm{HCO}-10 / \mathrm{CTAB}$ precipitate in water using vortexing for $15 \mathrm{~min}$ and aging overnight at $20^{\circ} \mathrm{C}$. Hexadecane $(15$ wt \%) was added to this cationized vesicle dispersion by vortexing and the mixture was sonicated for $15 \mathrm{~min}$ to produce the cationic three-phase emulsion. A conventional surfactant emulsion was also prepared using the same procedure with a $10 \mathrm{wt} \%$ CTAB solution used in place of the 10 wt \% mixed HCO-10/CTAB vesicle solution.

Figure 1 shows the relationship between the mole fraction of $\mathrm{CTAB}\left(X_{S}\right)$ in the CTAB/HCO-10 nanoparticles and their zeta potential. Formation of HCO-10 vesicles at $X_{S}=0$ was confirmed by TEM observation. The nanoparticle zeta potential increased with increasing $X_{S}$ from $-17 \mathrm{mV}$ to + $40 \mathrm{mV}$ and became constant when $X_{S} \geq 0.1$. Even when $X_{S}$ $=0.1$ it was found that vesicles could be formed. Therefore, the cationic vesicles formed at $X_{S}=0.1$ were adopted for use in the preparation of the cationic three-phase emulsion. We did not determine if vesicles formed when $X_{S}$ $>0.1$. 


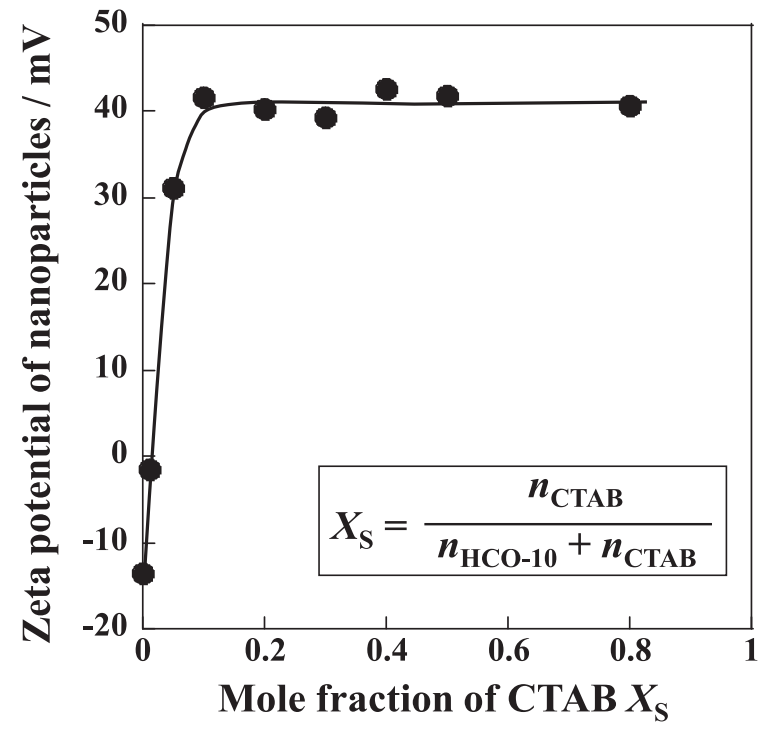

Fig. 1 Zeta potential vs. $X_{\mathrm{S}}$ for nanoparticles composed of mixed HCO-10 and CTAB.

\subsection{Adsorption test}

Adsorption experiments were carried out to determine whether or not the cationic three-phase emulsion adsorbs on various solid surfaces. The adsorption test procedure is described as follows.

A unit surface area $\left(1 \mathrm{~cm}^{2}\right)$ of the solid substrate was dipped in the three-phase emulsion; this was performed in varying concentrations of the three-phase emulsion adjusted by dilution with a given amount of water. After dipping in the emulsion the substrates were carefully dipped once in deionized water and then slowly removed. This rinsing procedure ensured the removal of droplets left on the substrate surface that were not adsorbed. Thus, the solid substrate with an adsorbed emulsion was obtained.

The adsorbed three-phase emulsion was characterized using weak sonication in a mixed solvent (40 mL 2-propanol, $10 \mathrm{~mL}$ ethanol, and $5 \mu \mathrm{L}$ decane) to remove the adsorbed emulsion from the solid substrate; the amount of hexadecane present was then determined quantitatively using gas chromatography.

The amount of the cationic surfactant-only emulsion that adsorbed was also determined using a similar procedure. In this case, we were careful not to dip the silicon substrate in the cationic surfactant emulsion for too long to avoid the formation of a double layer.

\section{RESULTS}

\subsection{Vesicle adsorption}

According to the adsorption theory discussed in Section 2, nanoparticles are expected to adsorb at water/solid-substrate interfaces regardless of whether or not the three-

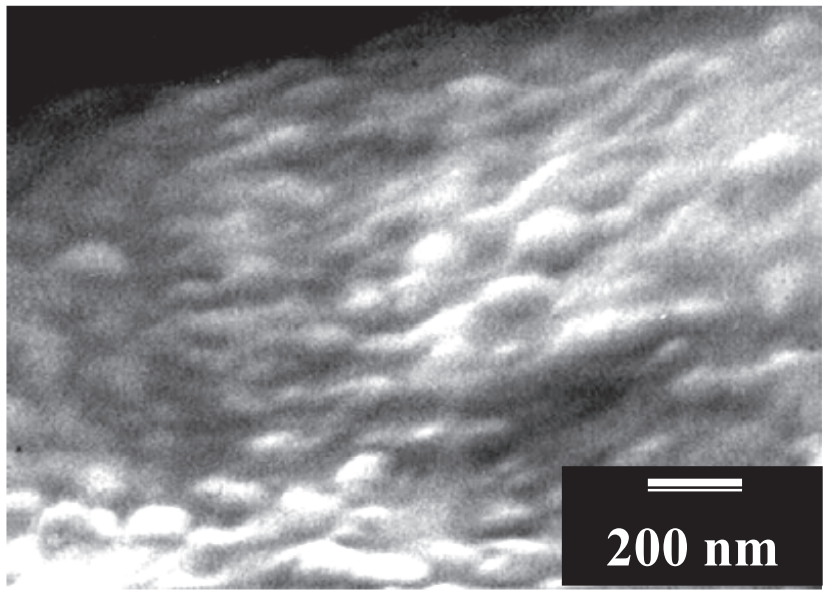

Photo 1 Scanning electron micrograph of HCO-10 vesicles adsorbed on a cellulose surface.

phase emulsion adsorbs on solid substrates in water. SEM was used to very this theory and determine whether the vesicles, acting as nanoparticles, adsorb at a water/solid substrate interface.

Cellulose fiber was dipped into the cationized HCO-10 vesicle dispersion and the mixture was stirred for $24 \mathrm{~h}$. After removal from the dispersion, carbon was deposited on the cellulose fiber surface under vacuum and SEM was then used to observe the surface. Photo 1 clearly shows that the vesicles are adsorbed on the cellulose surface. Therefore, the three-phase emulsion prepared with HCO-10/CTAB vesicles may be expected to adsorb on various solid substrates.

\subsection{Emulsion adsorption}

It is well known that surfactant molecules adsorb at various water/solid-substrates interfaces. However, it is very difficult to image adsorbed emulsions by TEM, as shown in Photo 2A. It is possible to directly observe the shape of the three-phase emulsion adsorbed on a solid surface by TEM using the negative-staining treatment described in Section 3.2, as shown in Photo 2B. Photo 2B shows that the three-phase emulsion maintains its structure both in water and an atmospheric environment; this is because there are no dependent components in the threephase emulsion system as discussed in Section 2.

Furthermore, we attempted to determine the speed of emulsion adsorption on the solid-substrate surface. The time dependence of the emulsion adsorption on a silicon plate was determined by measuring the weight of hexadecane on the silicon plate using a constant concentration of hexadecane (1.0 wt\%) and the same procedure described in section 3.2. Figure 2 shows the time course of emulsion adsorption. The amount of emulsion adsorbed increases with increasing time and eventually reaches at constant value, which appears to be an equilibrium value, after about three hours of adsorption time. Following this obser- 

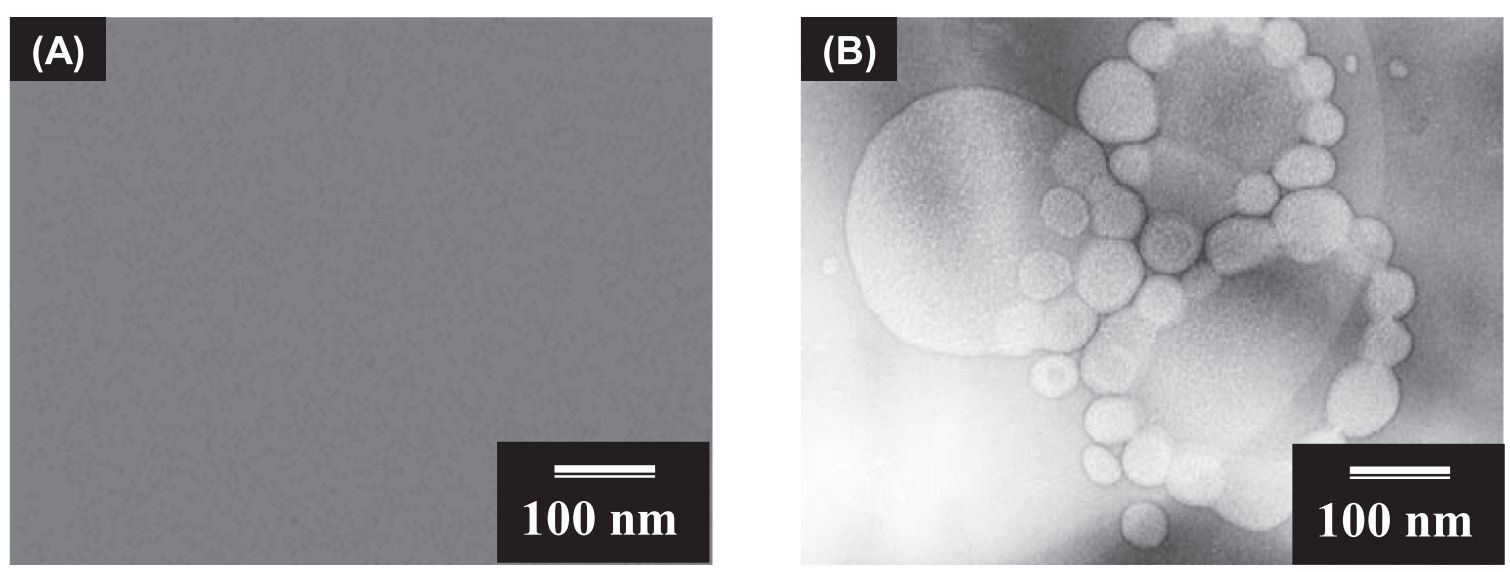

Photo 2 Transition electron micrographs of(A) a surfactant-only emulsion, and (B) a cationic three-phase emulsion on a copper surface.

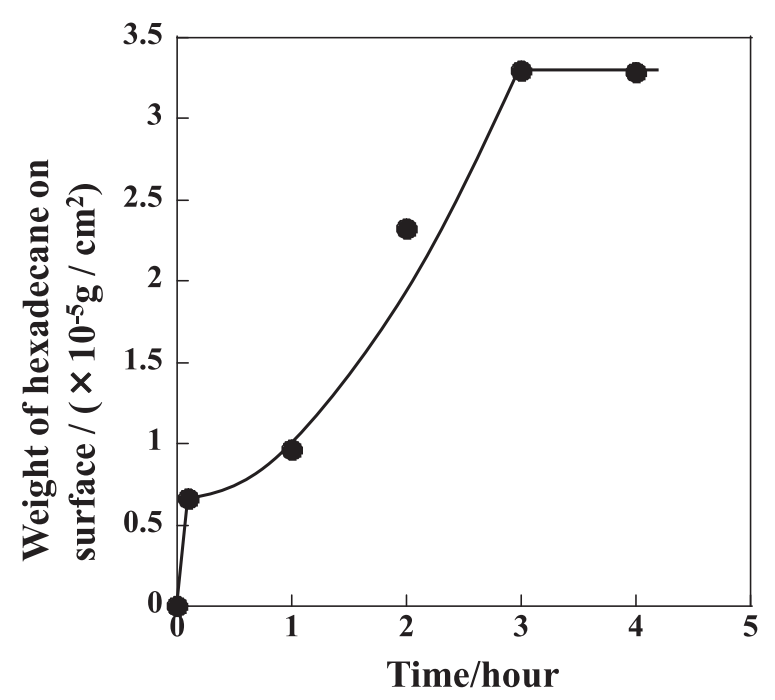

Fig. 2 Variation in the amount of hexadecane adsorbed from a $1 \mathrm{wt} \%$ hexadecane cationic emulsion on a silicon wafer surface over time at $20^{\circ} \mathrm{C}$.

vation, we tried to measure the emulsion adsorption isotherm, i.e. the relationship between the emulsion concen- tration and the amount of emulsion adsorbed at a constant temperature. This is discussed in Section 4.3.

We then investigated whether the three-phase emulsion adsorbs to a significant area of various solid substrates. The three-phase emulsion with a larger droplet size was prepared by homogenization(IKA, ULTRA-TURRAX T 25 digital) instead of sonication in order to observe the emulsion adsorption by optical microscopy. Photos $3 \mathrm{~A}$ and $3 \mathrm{~B}$ show optical micrographs of the three-phase emulsion adsorbed on a silicon wafer and a glass plate, respectively. The individual droplets are clearly visible and these findings confirm the three-phase-emulsion adsorption.

\subsection{Adsorption isotherm}

The adsorption of the cationic three-phase emulsion on various solid substrates was examined as a function of emulsion concentration. Figure 3 shows the adsorption isotherm of the hexadecane emulsions (three-phase and surfactant-only) on the silicon wafer surface at $20^{\circ} \mathrm{C}$. Scarcely a trace amount of the conventional surfactant emulsion adsorbed and its adsorption was independent of the hexadecane concentration. However, the amount of the
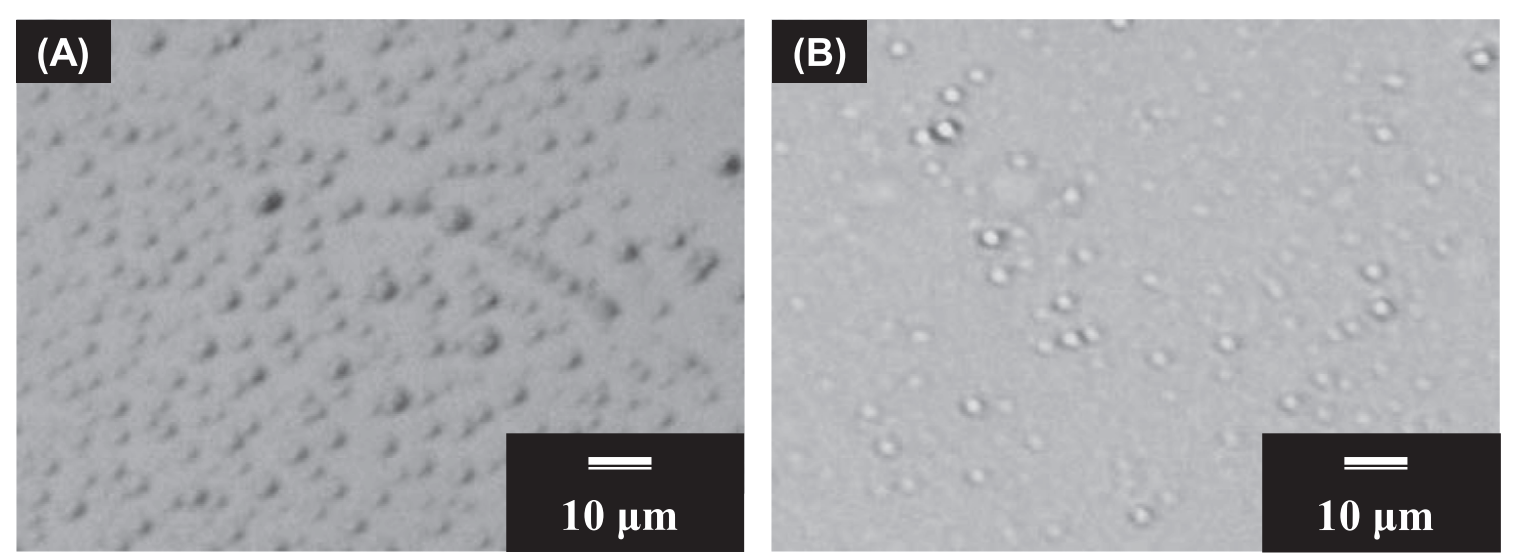

Photo 3 Optical micrographs of a cationic three-phase emulsion adsorbed on (A) a silicon wafer and (B) glass. 


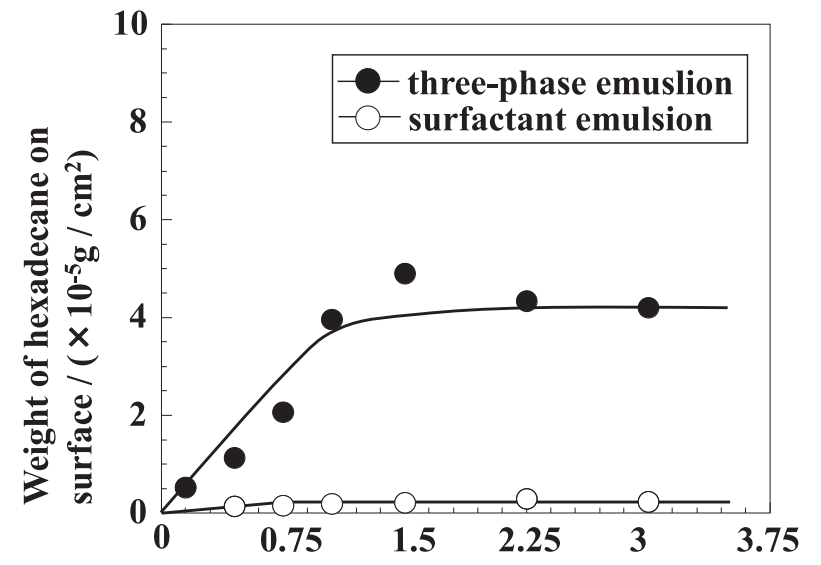

Concentration of hexadecane in emulsion / wt\%

Fig. 3 Concentration dependence of hexadecane adsorption from cationic three-phase and surfactant-only emulsions to a silicon wafer surface at $20^{\circ} \mathrm{C}$.

cationic three-phase emulsion that adsorbed increased with increasing hexadecane concentration and approached a constant value that may correspond to a saturation state. It is interesting to note that particle agglutination and piling into multiple layers does not seem to happen in the cationic three-phase-emulsion adsorption. The amount of hexadecane present on the silicon surface in the saturated state was about $4.09 \times 10^{-5} \mathrm{~g} / \mathrm{cm}^{2}$. The emulsion adsorption isotherm was also investigated on the glass and copper substrates using the same conditions as shown in Figs. 4 and 5, respectively, with similar tendencies observed for both emulsion types.

We also investigated the adsorption of the nonionic three-phase emulsion prepared by emulsification of hexadecane oil with the nonionic HCO-10 vesicle dispersion. In

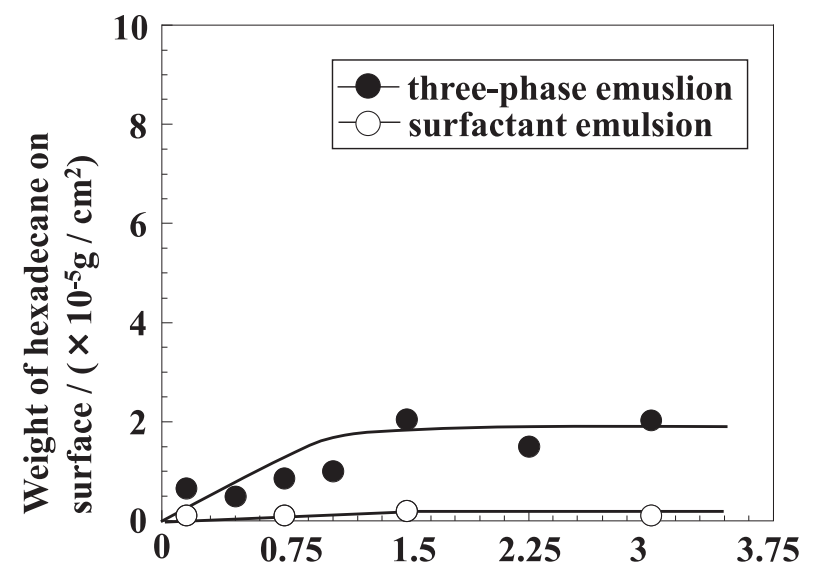

Concentration of hexadecane in emulsion / $\mathrm{wt} \%$

Fig. 4 Concentration dependence of hexadecane adsorption from cationic three-phase and surfactant-only emulsions to a glass surface at $20^{\circ} \mathrm{C}$.

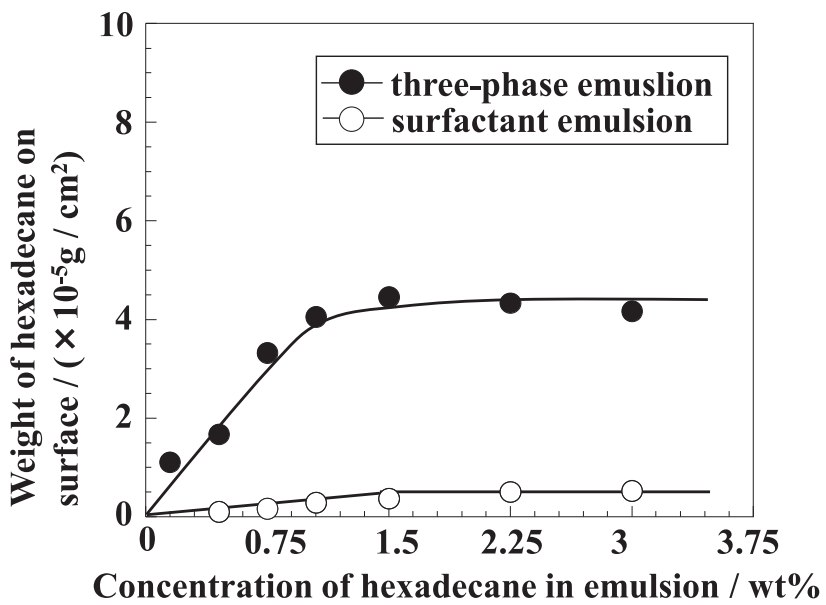

Fig. 5 Concentration dependence of hexadecane adsorption from cationic three-phase and surfactant-only emulsions to a copper surface at $20^{\circ} \mathrm{C}$.

this case, adsorption measurements on the various solid surfaces were carried out using with a hexadecane concentration of $3.0 \mathrm{wt} \%$ so as to give saturated adsorption of the nonionic HCO-10 emulsion system.

Zeta potentials of the emulsion droplets prepared with different emulsifiers were measured to account for ionic effects on the emulsion adsorption. The emulsion-droplet (adsorbate) zeta potentials are shown in Table 2, while those of the different solid surfaces (adsorbents) are shown in Table 1.

Table 3 shows the saturated-adsorption amounts of hexadecane for the different emulsions and surfaces as determined from Figs. 3, 4, and 5. The amount of the cationic three-phase emulsion adsorbed at saturation was much greater on all tested surfaces than both the surfactant-only emulsion and the nonionic three-phase emulsion. However, a significant amount of the nonionic three-phase emulsion (only HCO-10 vesicles used as the emulsifier) did adsorb on each of the solid substrates. This indicates that the adsorbability of the three-phase emulsion is not simply dependent on ionic interactions between the adsorbate emulsion droplets and the adsorbent solid surfaces. The adsorption mechanism is discussed further in Section 5.

\section{DISCUSSION}

Given the three-phase emulsion studied here can adsorb on solid substrates whilst maintaining its structure, significant amounts of various emulsified oils may be fixed on a desired solid surface. On the contrary, it appears that the conventional surfactant emulsion scarcely adsorbs even though it is cationic; however, the substrate was contaminated by hexadecane, because the emulsion cannot adsorb 
Table 2 Zeta potentials of the three different emulsions studied here in water.

\begin{tabular}{lcc}
\hline Preparation procedures & Emulsifiers & Zeta potential $/ \mathrm{mV}$ \\
\hline Three-phase emulsion & HCO-10 + CTAB & +40 \\
Surfactant emulsion & CTAB & +58 \\
Three-phase emulsion & HCO-10 & -17 \\
\hline
\end{tabular}

Table 3 Amount of hexadecane adsorbed from different emulsions at saturation on various solid substrates.

Amount of hexadecane adsorbed at saturation $\left(\times 10^{-5} \mathrm{~g} / \mathrm{cm}^{2}\right)$

\begin{tabular}{lc|cll}
\hline Preparation procedure & Emulsifiers & Silicon wafer & Glass & Copper \\
\hline Three-phase emulsion & HCO-10 + CTAB & 4.09 & 1.95 & 4.52 \\
Surfactant emulsion & CTAB only & 0.807 & 0.102 & 0.552 \\
Three-phase emulsion & HCO-10 only & 1.219 & 0.710 & 1.013 \\
\hline
\end{tabular}

on solid substrates whilst maintaining its structure. These experimental results are consistent with the predictions based on our theoretical analysis of emulsion adsorption discussed in Section 2. The trace amount of surfactantemulsion adsorption detected may be explained as follows. A monolayer of CTAB was quickly formed on the silicon wafer surface when the silicon wafer was dipped into the cationic CTAB emulsion; this rendered the silicon surface hydrophobic. The separated inner oil of the surfactant emulsion will then preferentially spread onto the hydrophobic surface. In other words, the presence of trace adsorbed hexadecane in the surfactant-emulsion system is just contamination of the substrate with the separated oil.

The absorbability differences presented in Table 3 were considered in light of the possible interactions between the three-phase emulsions and the solid substrates. Interaction potentials between particles were reported by Tajima ${ }^{35}$. In the case of the three-phase emulsion, the adhesion energy, $V(r)$, of the emulsifying particles to the oil droplets can be given by Equation (2) .

$$
V(r)=V_{\mathrm{A}}(r)+V_{\mathrm{E}}(r)+V_{\mathrm{S}}(r),
$$

where $V_{\mathrm{A}}(r)$ is the van der Waals attractive force, $V_{\mathrm{E}}(r)$ is the electrostatic interaction, and $V_{\mathrm{S}}(r)$ is the steric repulsion. Every term is given as a function of the distance, $r$, between the two materials. If $V_{\mathrm{A}}(r)$ is greater than the repulsive energy, expressed as the sum of $V_{\mathrm{E}}(r)$ and $V_{\mathrm{S}}(r)$, then the emulsifying nanoparticles will adhere to the oil droplets.

In the case of the cationic emulsifying particles, the negative zeta potential of the solid substrates results in a great electrostatic attraction $\left(V_{\mathrm{E}}(r)>0\right)$ between the particles and the substrates. By contrast, the negative zeta potential of the nonionic emulsifying particles results in an electrostatic repulsion $\left(V_{\mathrm{E}}(r)<0\right)$ between the emulsion and the solid substrates; thus, the nonionic three-phase emulsion was not expected to adsorb on the solid substrates. However, the amount of hexadecane adsorbed at saturation showed that the adsorbability of the nonionic threephase emulsion was superior to that of the cationic surfactant emulsion. The solid substrates can be classified in terms of their surface properties, with the surfaces of the silicon wafer and the copper being more hydrophobic than the glass surface, as indicated by their water contact angles. Thus, a large quantity of water will be fixed on the glass surface resulting in a great steric repulsion $\left(V_{\mathrm{S}}(r)<\right.$ $0)$.

Similarly, this equation is effective for understanding the adsorption interactions between the emulsifying particles, fixed on the oil droplets, and the solid substrate surfaces; the properties of the emulsifying nanoparticles should be viewed as an independent phase that does not depended on every given state as described in Section 2. In other words, the nanoparticles used here may act as a kind of particle binder between the oil droplets and the solid surface as illustrated in Scheme 1. Therefore, the adsorption behavior cannot be understood simply by considering the long-range electrostatic interaction and the wettability of the solid surface; the attractive energy $\left(V_{\mathrm{A}}(r)\right)$ in Equation (2) must also be considered.

The coverage of each surface by the adsorbed emulsions was estimated from the results presented in Table 3 . It is practically very important to know the distribution of the adsorbed emulsion on the solid surface. The average droplet size in each emulsion was measured by DLS (dynamic light scattering) and found to be about $1.0 \mu \mathrm{m}$ and $1.2 \mu \mathrm{m}$ for the emulsions stabilized with cationized and non-cationized, respectively, HCO-10 vesicles. Therefore, we may easily estimate the surface coverage values for each solid substrate, i.e. the distribution of the adsorbed emulsions. These values are presented in Table 4 and show that the amount of oily substance from the cationic three- 
Table 4 Surface coverage of cationic and nonionic three-phase emulsions on various solid substrates.

\begin{tabular}{lc|ccc}
\hline Charge & Particle size $/ \mathrm{nm}$ & Silicon wafer $/ \%$ & Glass $/ \%$ & Copper $/ \%$ \\
\hline Cation & 1000 & 71.9 & 34.3 & 79.4 \\
Nonionc & 1200 & 17.8 & 10.4 & 14.8 \\
\hline
\end{tabular}

phase emulsion adsorbed on the three surfaces is about $200-400 \times$ greater than that of a closed-packed monolayer, using the saturated adsorption amount $\left(3 \times 10^{-10} \mathrm{~mol} / \mathrm{cm}^{2}\right)$ of the cationic molecule reported by Nakamura and Tajima ${ }^{36)}$.

These findings on emulsion adsorption are expected to apply to the adsorption of emulsified functional oils such as anti-fungal agents, agricultural chemicals, and pest repellents. Furthermore, we have shown that bath agents composed of the three-phase emulsion are practically effective for retaining warmth ${ }^{37)}$ and detergents using the threephase emulsification can be applied to the removal of residual oil on solid surfaces ${ }^{38,39}$.

The findings of this study can be summarized as follows. Since hexadecane was the common inner oil in all emulsions, the adsorbability of the three-phase emulsions did not depend on the properties of the inner oil, rather it depended on the emulsifying particles. The combination of the emulsifying-particle charge and the surface properties of the solid substrates, affected the interaction energy $V$ $(r)$, which dictated the differences seen in the amount of emulsion adsorbed in each system. Moreover, through analysis of the emulsifying-particle (vesicle) adsorption energy $V(r)$ it was found that both the ionic and non-ionic three-phase emulsions adsorbed irreversibly to the solid substrates.

\section{CONCLUSION}

The following points summarize the main achievements and conclusions of this study:

1) The amount of the cationic three-phase emulsion that adsorbed increased with increasing hexadecane concentration and eventually reached a saturation state, similar to molecular adsorption. The three-phase emulsion adsorbed to silicon, glass, and copper substrates while maintaining its structure.

2) The novel mechanism of three-phase-emulsion adsorption on the solid substrates was explained using the theory of irreversible adhesion. The combination of the emulsifying-particle charge and the surface properties of the solid substrates determined the interaction energy $V(r)$ between the emulsion droplets and the solid substrates.

\section{ACKNOWLEDGMENT}

Mr. Y.E. would like to express his sincere appreciation to the Mizuho Industrial Co., Ltd. and the late Dr. Takagi for giving him the opportunity to study chemistry at Kanagawa University.

We would also like to thank Editage (www.editage.jp) for English language editing.

\section{References}

1) Bangham, A.D.; Horne, R.W. Negative staining of phospholipids and their structural modification by surfaceactive agents as observed in the electron microscope. J. Mol. Biol. 8, 660-668(1964).

2) Benson, A.A. On the orientation of lipids in chloroplast and cell membranes. J. Am. Oil Chem. Soc. 43, 265270 (1966).

3) Zhang, M.; Meng, L.; Cao, X.; Jiang, M.; Yi, T. Morphological transformation between three-dimensional gel network and spherical vesicles via sonication. Soft Matter 8, 4495-4500 (2012).

4) Brito, R.O.; Marques, E.F. Neat DODAB vesicles: Effect of sonication time on the phase transition thermodynamic parameters and its relation with incomplete chain freezing. Chem. Phys. Lipids 137, 18-28 (2005).

5) Santucci, E.; Carafa, M.; Coviello, T.; Murtas, E.; Riccieri, F.M.; Alhaique, F.; Modesti, A.; Modica, A. Vesicles from polysorbate 20 and cholesterol: A simple preparation and a characterization. STP Pharma Sci. 6, 29-32 (1996).

6) Turanek, J.; Kasna, A.; Zaluska, D.; Neca, J. Preparation of sterile liposomes by proliposome-liposome method. Methods Enzymol. 367, 111-125(2003).

7) Yamaguchi, S.; Tsuchiya, K.; Sakai, K.; Abe, M.; Sakai, $\mathrm{H}$. Preparation of nonionic vesicles using the supercritical carbon dioxide reverse phase evaporation method and analysis of their solution properties. $J$. Oleo Sci. 65, 21-26 (2016).

8) Wang, L.; Pileni, M. Encapsulation of Zwitterionic Au Nanocrystals into Liposomes by Reverse Phase Evaporation Method: Influence of the Surface Charge. Langmuir 32, 12370-12377 (2016).

9) Niu, W.; Feng, S.; Zhou, X.; Zhang, W.; Liu, Q.; Kong, L. Preparation of black rice bran anthocyanin liposomes by reverse-phase evaporation combing with $\mathrm{pH}$ gradi- 
ent. Shipin Gongye Keji 36, 238-242, 247 (2015).

10) Chen, S.; Lu, Q.; Liu, X.; Yu, C.; Chen, L.; Xie, F. Preparation and characterization of metronidazole liposome by supercritical reverse phase evaporation method. Huaxue Gongcheng (Xi'an, China) 41, 58-61 (2013).

11) Oliveira, C.B.; Rigo, L.A.; Rosa,L.D.; Gressler, L.T.; Zimmermann, C.E.; Ourique, A.F.; DASilva, A.S.; Miletti, L.C.; Beck, R.C.; Monteiro, S.G. Liposomes produced by reverse phase evaporation: in vitro and in vivo efficacy of diminazene aceturate against Trypanosoma evansi. Parasitology 141, 761-769 (2014).

12) Enomoto, Y.; Imai, Y.; Tajima, K. A preferable method for the formation of vesicles from lamellar liquid crystals using chemical additives. J. Oleo Sci. 66, 297-306 (2017).

13) Sharma, M.; Bhowmick, R.; Gappa-Fahlenkamp, H. Drug-loaded nanoparticles embedded in a biomembrane provide a dual-release mechanism for drug delivery to the eye. J. Ocul. Pharmacol. Ther. 32, 565573 (2016).

14) Bravo-Osuna, I.; Andres-Guerrero, V.; Pastoriza A.P.; Molina-Martinez, I.T.; Herrero-Vanrell, R. Pharmaceutical microscale and nanoscale approaches for efficient treatment of ocular diseases. Drug Delivery Transl. Res. 6, 686-707 (2016).

15) Singh, S.; Vardhan, H.; Kotla, N.G.; Maddiboyina, B.; Sharma, D.; Webster, T.J. The role of surfactants in the formulation of elastic liposomal gels containing a synthetic opioid analgesic. Int. J. Nanomed. 11, 14751482 (2016).

16) Chen, H.; Tham, H.P.; Ang, C.Y.; Qu, Q.; Zhao, L.; Xing, P.; Bai, L.; Tan, S.Y.; Zhao, Y. Responsive prodrug selfassembled vesicles for targeted chemotherapy in combination with intracellular imaging. ACS Appl. Mater. Interfaces 8, 24319-24324(2016).

17) Saitejaswi, R.; Swapna, S.; Madhu, B.A.; Bakshi, V. Formulation and evaluation of lornoxicam loaded transfersome gel. Eur. J. Biomed. Pharm. Sci. 3, 144-150 (2016).

18) Singla, S.; Harjai, K.; Raza, K.; Wadhwa, S.; Katare, O. P.; Chhibber, S. Phospholipid vesicles encapsulated bacteriophage: A novel approach to enhance phage biodistribution. J. Virol. Methods 236, 68-76 (2016).

19) Tajima, K.; Imai, Y.; Nakamura, A.; Koshinuma, M. Solubilization of hydrocarbons into a bilayer assembly of dimyristoylphosphatidylcholine in water. Colloids Surf. A 155, 311-322(1999).

20) Tajima, K.; Imai, Y.; Horiuchi, T. Jpn. Pat., 3855203 (2006).

21) Tajima, K.; Imai, Y.; Maekawa, S. Jpn. Pat., 5618269 (2014).

22) Tajima, K.; Imai, Y.; Funatsu, R. Jpn. Pat., 5181354 (2013).

23) Tajima, K.; Imai, Y. Jpn. Pat., 5344277(2013).
24) Tajima, K.; Imai, Y. Jpn. Pat., 5409991 (2013).

25) Tajima, K.; Imai, Y. Jpn. Pat., 4552198(2010).

26) Imai, Y.; Toyoda, K.; Koshinuma, M.; Tajima, K. Experimental confirmation for structural validity of threephase emulsion. in the 52nd Annual Meeting of the Japan Oil Chemists' Society, Miyagi, Abstracts. p. 155 (2013).

27) M. D. Berthiaume; J. Jachowicz. The effect of emulsifiers and oil viscosity on deposition of nonionic silicone oils from oil-in-water emulsions onto keratin fibers. $J$. Colloid Interface Sci. 141, 299-315(1991).

28) Gould-Boeder, J.M.; Malec, A.D.; Figley, T.M. Utilizing solvent blends to maximize green characteristics of emulsifiable concentrate formulations. Abstracts of Papers, 239th ACS National Meeting, San Francisco, CA, United States, pp. 21-25(2010).

29) Friloux, K.M.; Tann, R.S. Preparation and evaluation of concentrated emulsions of agrichemicals. ASTM Special Technical Publication. pp. 59-72(1993).

30) Shang, W.; Yuan, T.; Wang, F.; Hu, J.; Tu, W. Synthesis and performance of organic silicone modified phosphorus-containing styrene acrylic anti-rust emulsion. Reguxing Shuzhi 29, 33-38(2014).

31) Fang, X; Chen, S. Synthesis and application of acrylate anti-rust emulsion modified by dimer acid with selfcrosslinking core-shell structure. Dangdai Huagong 42, 574-576 (2013).

32) Horiuchi, T; Tajima, K. Thermal properties of aqueous vesicle dispersion formed with poly (oxyethylene) hydrogenated castor oil. Yukagaku 41, 1197-1202 (1992).

33) Sun, C.; Ueno, M. Formation and properties of HCO-10 vesicles. Colloid Polym. Sci. 275, 155-161 (1997).

34) Johnson, S.M.; Bangham, A.D.; Hill, M.W.; Korn, E.D. Single bilayer liposomes. Biochim. Biophys. Acta 233, 820-826 (1971).

35) Tajima, K.; Koshinuma, M.; Nakamura, A. Steric repulsion of polyoxyethylene groups for emulsion stability. Colloid Polym. Sci. 270, 759-767(1992).

36) Nakamura, A.; Tajima, K. Surface activity of complexes formed with N-dodecyl- $\beta$-alanine and copper (II) Ion in Aqueous Solution. Bull. Chem. Soc. Jpn. 61, 38073812 (1988).

37) Tajima, K.; Imai ,Y. et al. Fragrance J. 2016-5, 18-23 (2016).

38) Enomoto, Y.; Takagi, K.; Imai, Y.; Tajima, K. Washing properties of silicone oils contaminated on the emulsifying equipment by the three-phase emulsification: dimethyl silicone oils with high viscosity. in the 97th CSJ Annual Meeting, Chiba, Abstracts. 2 PC-051 (2015).

39) Enomoto, Y.; Takagi, K.; Imai, Y.; Tajima, K. Thermodynamic analysis of the transition of liquid crystals from lamellar to vesicular phase. J. Oleo Sci. 66, 375-382 (2017). 